\title{
ESPACIO, POETOLOGÍA Y CORPORALIDAD EN BORDERLANDS / LA FRONTERA DE GLORIA ANZALDÚA. DINÁMICAS FRONTERIZAS ENTRE MODOS DE EXPRESIÓN GENÉRICOS Y MODOS PERCEPTIVOS
}

Space, Poetics and Corporality in Borderlands/La Frontera by Gloria Anzaldúa. Border Dynamics between Literary Genres and Modes of Perception

ANNE BRÜSKE

Universidad de Heidelberg / Universidad de Ratisbona (Alemania) anne.brueske@uni-heidelberg.de ORCID: https://orcid.org/0000-0002-8477-7751

INÉZ MARIA WELLNER

Universidad de Heidelberg (Alemania)

inez-maria.wellner@rose.uni-heidelberg.de

ORCID: https://orcid.org/0000-0003-0176-2667 


\section{Resumen}

En este artículo, se propone una relectura de la obra canónica Borderlands/La Frontera de la pensadora chicana Gloria Anzaldúa, desde la perspectiva de su poetología y de los espacios que el texto produce a través de su hibridez genérica y de la relevancia de la corporalidad del sujeto. Asimismo, en el texto se crea no solo una experiencia multidimensional sino más bien un espacio de comunicación entre el texto y sus audiencias, y un diálogo intersubjetivo entre 'las nuevas Mestizas' y lectores y lectoras que no participen en esta experiencia. Para sostener esta línea de argumentos, recurrimos a un close reading del texto como obra de arte combinado con una versión descolonizada del modelo fenomenológico del espacio desarrollado por Henri Lefebvre en La production de l'espace (1974).

\section{Palabras clave}

Borderlands/La Frontera, espacio, poetología, corporalidad, Gloria Anzaldúa

\section{Abstract}

This article proposes a new reading of the canonical work Borderlands/La Frontera (1987) by Chicana thinker Gloria Anzaldúa in terms of its poetics and the spaces the text produces through its generic hybridity and its accentuation of subjective corporeality. We argue that the text creates not only a multidimensional experience but rather a space of communication between the text and its audiences as well as an intersubjective dialogue between 'the new Mestizas' and readers who do not share this experience. Our analysis draws on a close reading of the text as a piece of art along with a decolonized version of the phenomenological model of space developed by Henri Lefebvre in La production de l'espace (1974).

\section{Keywords}

Borderlands/La Frontera, Space, Poetology, Corporality, Gloria Anzaldúa

Borderlands/La Frontera: The New Mestiza (1987) representa una de las obras más conocidas de la literatura fronteriza que ha surgido en la zona atravesada desde el siglo XIX por la frontera norte/suroeste entre EE. UU. y México. ${ }^{1}$ Se trata de uno de los textos centrales de la literatura de autoras chicanas que ganó visibilidad en el suroeste de EE. UU. a partir los años ochenta con intelectuales como Gloria Anzaldúa, Sandra Cisneros, Ana Castillo o Norma Alarcón. ${ }^{2}$ Se inició, en ese momento, en la literatura y en la producción artística fronteriza un nuevo diálogo sobre la materialidad y el simbolismo de la frontera y del contacto cultural conflictivo caracterizado por relaciones de poder

\footnotetext{
${ }^{1}$ Para un breve panorama de la literatura de esta zona geográfica, véase Heide, 2004: 19-25; Gewecke, 2012.

Hay que destacar igualmente que una gran cantidad de autores y autoras deploran la hegemonía estadounidense en el campo literario de la frontera (Abrego, 2011: 50-51) o subrayan las diferentes perspectivas (Bandau, 2020: 21-23).

2 Para una evaluación crítica de la literatura hecha por mujeres escribiendo desde la frontera, véase Castillo y Socorro Tabuenca, 2002.
} 
entre poblaciones angloamericanas, mexicanas y mestizas convirtiendo Borderlands/La Frontera en un texto seminal de los estudios fronterizos.

¿Por qué leer a Anzaldúa hoy? ¿Por qué revisitar esta obra sumamente canónica a principios de la tercera década de este nuevo milenio? ¿Por qué recurrir a un nuevo close reading de este texto a pesar de voces críticas que deploran su proximidad con el campo poscolonial anglófono o que ven en su canonización por la academia anglófona otro (neo)colonialismo estadounidense (Ábrego, 2011; Vila, 2000) $?^{3}$ En estas últimas décadas, después de la publicación de Borderlands/La Frontera, las fronteras y las materialidades fronterizas han ganado peso en el discurso público interamericano y mundial, sobre todo frente a la precaria situación actual de un número inmenso y creciente de migrantes globales que va agravándose. No menos importantes son las heurísticas 'pos pos' que, frente a esta crisis, quieren ofrecer nuevos instrumentos y teorías para analizar los efectos de la migración sobre sujetos y procesos culturales. Estos se distancian cada vez más del 'momentum posmoderno' de las décadas de los ochenta y noventa (Ette, 2014), cuando nació el entusiasmo algo desmaterializado de Néstor García Canclini en Culturas híbridas (1990) precisamente en relación con la frontera norte/suroeste entre México EE. UU. como laboratorio posmoderno de "heterogeneidad multitemporal" (García Canclini, 1990: 15, 72). ${ }^{4}$

Pensamos que vale la pena volver al texto complejo de Anzaldúa, ya que funciona como una obra artística compleja y un análisis sociocultural sólido y que entrelaza elementos culturales, temporales, lingüísticos y espaciales diferentes. También genera, de este modo, "heterogeneidades multitemporales". Sin embargo, a diferencia de Canclini (y de Homi K. Bhabha), favorece un análisis y una actitud política con base en lo material, en el impacto de políticas culturales, lingüísticas y laborales sobre las condiciones de vida de los sujetos fronterizos. Asimismo, busca estrategias para re-escribir la Historia y las historias de los sujetos de los borderlands no solo para darles visibilidad al modo en que lo han hecho recientemente proyectos de arte virtual como Carne y Arena de Alejandro González Iñárritu $(2017-2018)^{5}$ o la instalación y performance Transborder Immigrant Tool del grupo The Electronic Disturbance Theater 2.0 establecido por Ricardo Domingo (2008-2010), ${ }^{6}$ sino también para habilitarlos e incitar a sus lectores y lectoras a entrar en un diálogo intersubjetivo con estos últimos. En este sentido, Borderlands / La Frontera se distancia de una potencial (re)victimización y despolitización de los sujetos fronterizos por el arte o, más aún, por las industrias culturales que al hacer espectáculo del dolor y de la muerte exhiben destinos humanos precarios por fines de entretenimiento y de beneficio

\footnotetext{
${ }^{3} \mathrm{Si}$ es verdad que Anzaldúa se acerca con su noción de los borderlands a la idea del tercer espacio desarrollada por Homi K. Bhabha (Ábrego, 2011: 48-49) y que el discurso crítico estadounidense se ha apropiado en cierta manera del texto como paradigma universal de los Postcolonial Studies, también es cierto que otras publicaciones destacan la filiación mexicana de la obra (Stehn y Alessandri, 2020).

${ }^{4}$ Véase también Ábrego, 2011: 48-50 y Giménez, 2005: 26-27.

${ }^{5} \mathrm{El}$ proyecto "Carne y Arena" es un híbrido de exhibición artística, reconstrucción de eventos históricos y simulación con realidad virtual del cruce entre la frontera de México y Estados Unidos. Se trata de un proyecto que se exhibió simultáneamente entre 2017 y 2018 en el Los Angeles en County Museum of Art (LACMA) y en el Centro Cultural Universitario Tlatelolco en Ciudad de México, donde los visitantes pudieron "experimentar" el cruce fronterizo a través de un sistema de simulación de realidad virtual. Aunque el proyecto dio la posibilidad de ponerse en la situación de los y las migrantes a nivel acústico, táctil y visual, se debe resaltar que esta experiencia fue limitada en el tiempo de la visita y que solo una élite intelectual y financiera tuvo el privilegio de acceder a las entradas. Por ello, se crea un abismo existencial entre sujetos representados (o reemplazados) y visitantes que plantea importantes preguntas éticas sobre la relación entre lo representado, la forma de representación y la recepción frente a la miseria humana.

${ }^{6}$ Véase, por ejemplo, la entrevista de Markus Heide con Ricardo Domínguez en la que aborda la cuestión de la ética de este tipo de arte transfronterizo (Heide, 2021). Otros proyectos como La región de los pantalones transfronterizos (The Region of Transborder Trousers) (2004-2005) del grupo Torolab ubicado en Tijuana (Radlwimmer, 2020: 8-9) se posicionan igualmente en favor de los sujetos migrantes prestándoles ayuda en el cruce de la frontera e intentando resignifar el duelo de los y las migrantes. Sin embargo, se trata de proyectos de doble filo puesto que algunos de ellos revelaron por un video cartográfico los movimientos rastreados por GPS en la zona fronteriza entre Tijuana y San Diego y, por eso, rutas del cruce a través de una frontera fuertemente vigilada.
} 
financiero. Al mismo tiempo, problematiza la idea de que se pueda vivir la experiencia del Otro de manera inmediata a través de su mera representación.

En definitiva, en Borderlands/La Frontera - como en la antología publicada anteriormente y coeditada con Cherríe Moraga: This Bridge Called My Back. Writings by Radical Women of Color (1981)— se manifiesta un punto de vista ético decisivo que va más allá del ejemplo de la frontera norte/suroeste, sus cruces peligrosos y sus choques culturales. El presente artículo pone énfasis en la relación íntima entre espacio, poetología y corporalidad en Borderlands/La Frontera para acentuar en qué medida el texto formula una intervención práctica y una concepción teórica compleja en dicho conjunto. Nos importa darle nueva visibilidad a este texto como respuesta ética a proyectos artísticos contemporáneos como Carne y Arena o más aun películas comerciales de carácter sensacionalista como Sin nombre (2009), Babel (2006) o La Frontera (2014); y mostrar que el pensamiento de Anzaldúa sigue estando vigente en este nuevo milenio y en el contexto de las migraciones a escala global.

Borderlands/La Frontera se ha vuelto una obra canónica de los Estudios Fronterizos, de las Chicana Studies y, más allá, del Feminism of Color y de los Estudios Poscoloniales, ${ }^{7}$ ya que negocia temas del espacio material, metafórico y del contacto cultural situándose en la intersección de varios ejes que definen la posición de un sujeto dentro una sociedad histórica. A un primer nivel, el texto trata la situación de vida de los Chicanos, sobre todo las Chicanas, en la región fronteriza entre el Suroeste de EE. UU. y México de los años ochenta, territorio en el que coexisten, se entrechocan y, a veces, conviven diferentes culturas (estadounidense, mexicana, católica, indígena), diferentes lenguas (español, inglés, spanglish) y diferentes expectativas frente al papel de 'la mujer'. Muestra los efectos violentos de exclusión y marginalización de estas demarcaciones fronterizas concretas entre religiones, etnias, comunidades lingüísticas, clases y sexos en tanto se abren perspectivas para acercarse a situaciones comparables. Más allá del nivel temático, el nivel textual formal refleja las posibilidades y límites de contactos culturales o intersubjetivos no solo bajo condiciones de hegemonía colonial y neocolonial, sino de un modo general. Este entrelazamiento se expresa a través de una pluralidad estilística, una yuxtaposición de diversos géneros dentro de la obra, que existe de manera paralela a la acentuación del papel decisivo del cuerpo humano, no solo en la producción cotidiana de espacios y culturas fronterizos, sino también en la producción textual de estos espacios. Por lo tanto, nos preguntamos: ¿qué potencial de creación del espacio, en una situación de contacto fronterizo marcado por relaciones de poder (pos)coloniales, se está realizando tanto en las prácticas textuales como en las prácticas corporales? ¿Qué papel tiene la poetología de la obra en esta problemática? ¿Por qué el texto insiste tanto en el cuerpo y en la corporalidad para la creación de dicho espacio?

\section{Estrategias poetológicas}

\footnotetext{
${ }^{7}$ De hecho, el texto de Anzaldúa es citado por un gran nombre de intelectuales pos o decoloniales, por ejemplo: Homi K. Bhabha, Gayatri Spivak, Enrique Dussel, Mary Louise Pratt, Aníbal Quijano y Walter Mignolo. En la academia estadounidense, la obra de Anzaldúa ha servido de inspiración para desarrollar líneas de pensamiento como el border thinking o para consolidar áreas como el Feminism of Color (Keating, 1996 y 2005) o los Queer Studies; mientras que en la academia mexicana (Socorro Tabuenca, 1995; Vila, 2000) se ha formulado una crítica aguda de la perspectiva demasiado estadounidense, escapista y anacronista del proyecto de Anzaldúa (Castillo, 2006: 260-261). Para un resumen de la discusión crítica de la incorporación de la obra en diferentes tradiciones discursivas (y de su apropiación o "tokenización” en los Transnational American Studies estadounidenses), véase Castillo, 2006.
} 
En la amplia recepción crítica de Borderlands/La Frontera: The New Mestiza, hay dos observaciones que se mencionan de manera regular al describir las características de la obra (y que denotan cierta tensión entre sî): la existencia de una hibridez genérica ${ }^{8}$ y el predominio del modo de habla autobiográfico. ${ }^{9} \mathrm{Si}$ empleamos la heurística de interpretación contextual, la hibridez genérica alude a un modo de habla auto-etnográfico o de "autohistoria" que escenifica un pluralismo de identidades y, por tanto, remite a la construcción de una identidad poscolonial y feminista, en la que se establece una vOz propia como representación alternativa dentro de un discurso hegemónico masculino, heterosexual y occidental-eurocéntrico. ${ }^{10} \mathrm{El}$ texto, desde dicho punto de vista, es la escenificación de un sujeto que no es idéntico sino plural. Por ende, en esta interpretación, la obra de arte representa y exhibe una experiencia de un sujeto para constituir cierta voz dentro de un discurso, lo cual es en sí un acto político. No obstante, sería reduccionista implementar una noción de la obra de arte concebida como un mero instrumento de representación y obviar así, en cierta medida, su carácter artístico, es decir, su capacidad de producir una variedad de imágenes y de sentidos. Bornstein-Gómez indica: "As a writer, Anzaldúa understands the power inherent in the discursive construction of identities and histories and the communication of cultural experience. In this context, art becomes ritual" (2010: 53).

En el presente análisis proponemos ampliar las lecturas contextuales por una heurística poetológica, que tematiza la obra artística en sí y que destaca cómo el modo autobiográfico está siendo subvertido a favor de la constitución de un entendimiento del texto literario como espacio de comunicación entre una obra y su público lector. Este punto de vista también se localiza dentro de la propuesta de Anzaldúa más allá de Borderlands/La Frontera cuando uno se da cuenta de la importancia del papel de la pedagógica en general para la autora. ${ }^{11}$ El texto se muestra así como una materialidad concretizada a través de la voz que lo lee, del sujeto lector, quien lo experimenta a nivel mental y físicocorporal. De este modo, el texto subraya su carácter material como espacio de comunicación. La hibridez genérica puede ser entendida como una estética compuesta de diferentes estilos. Esta estética establece un intento de comunicar de una manera que consigue satisfacer diferentes dimensiones de experiencia de las realidades individuales y colectivas.

\section{El texto como espacio de comunicación}

Borderlands/La Frontera tiene un prefacio, que acorde con su función, comenta lo que "va a venir". Aquí el modo autobiográfico es subvertido a favor de la constitución de una situación de comunicación muy particular entre la obra y el lector y la lectora. Un close reading del prefacio muestra que la misma obra dificulta una lectura únicamente auto-etnográfica. Inicialmente se encuentran pruebas de la identidad entre autora explícita, instancia narrativa y figura. En la portada se dice "Gloria Anzaldúa", en el prefacio se adopta un discurso que recupera la memoria en primera persona: "[...] this book then speaks of my existence" (Anzaldúa, 1999: 19). ${ }^{12} \mathrm{El}$ pacto autobiográfico según Lejeune parece estar firmado (Lejeune, 2001) ${ }^{13} \mathrm{El}$ yo se da a conocer como mujer de frontera: "I am a border woman" (Anzaldúa, 1999: 19). Una existencia con experiencias dolorosas, causadas por la vida en la frontera. Este yo, sin embargo, se ve transformado en la categoría de mestiza en la frase siguiente:

\footnotetext{
8 Véase Garza, 2003: 196.

${ }^{9}$ Véase Keating, 1996.

10 Arturo Aldama entiende la voz narrativa de Borderlands/La Frontera como una "expresión autoetnográfica" — de acuerdo con Mary Louise Pratt— y enfatiza su potencial en procesos de reescritura de subjetividades marginalizadas (véase Aldama, 2001: 95; Pratt, 1992: 7). Para la noción de autohistoria en la obra de Anzaldúa, véase también Dahms, 2021.

${ }^{11}$ Véase, por ejemplo: Nuño, 2020 y Barrera, 2020.

$12 \mathrm{Al}$ lector se le ofrece leer el texto tanto como un enunciado personal como uno sobre la realidad (Langer, 2007: 179-180).

${ }^{13}$ Según Philippe Lejeune (2001), una autobiografía tiene como base la firma de un pacto (explícito o implícito) entre las instancias del lector y del autor; lo narrado corresponde a la realidad que las dos instancias comparten.
} 
"However, there have been compensations for this mestiza, and certain joys. Living on borders and in margins, keeping intact one's shifting and multiple identity and integrity, is like trying to swim in a new element, an 'alien' element. There is an exhilaration in being a participant in the further evolution of humankind, in being 'worked' on” (Anzaldúa, 1999: 20).

El pronombre demostrativo "this" paraleliza la instancia narrativa en primera persona a través de la categoría de "mestiza". Nadar en un elemento ajeno es la imagen utilizada para el proceso de aprendizaje, planteado por el reto de (sobre-)vivir en la frontera. Este proceso, sin embargo, no es un proceso individual, sino parte del desarrollo de toda la humanidad. Un desarrollo que describe una utopía, dado que se asocia a la alegría, vale decir: es un proceso deseado. Su punto final parece ser la existencia de individuos nuevos descritos como "new mestizas", como muestran las últimas frases del prefacio: "But we Chicanos no longer feel that we need to beg entrance, that we need always to make the first overture [...]. Today we ask to be met halfway. This book is our invitation to you - from the new mestizas" (Anzaldúa, 1999: 20).

Si consideramos lo anterior, se aclara la situación de comunicación específica de Borderlands/La Frontera. Tanto la instancia narrativa como el objeto narrativo están sujetos a cierto dinamismo semántico introducido en el prefacio. Hemos visto una abstracción de lo más específico hasta lo más general, pasamos del 'yo' a la categoría de 'mestiza'. Eso también ocurre con el objeto narrativo, la frontera y la vida en el Borderland:

The actual physical borderland that I'm dealing with in this book is the Texas-U.S. Southwest/Mexican border. The psychological borderlands, the sexual borderlands and the spiritual borderlands are not particular to the Southwest. In fact, the Borderlands are physically present wherever two or more cultures edge each other, where people of different races occupy the same territory, where under, lower, middle and upper classes touch, where the space between two individuals shrinks with intimacy. (Anzaldúa, 1999: 19)

Aunque al principio el objeto narrativo es el "actual physical borderland that I'm dealing with", este "borderland" se transforma en el concepto "Borderland" con mayúscula, un espacio que puede ser experimentado por cada una y cada uno. Y esta también es la condición previa para la parte final del prefacio. Si la experiencia del "Borderland", como espacio donde uno tiene que mantener una pluralidad de existencias e identidades, forma el punto de partida del proceso de desarrollo de la humanidad, tiene que ser posible que toda la humanidad o, más concretamente, cada individuo pueda experimentarla hasta convertirse en un tipo de mestiza. En tanto se afirma que el libro "is our invitation to you -from the new mestizas" (Anzaldúa, 1999: 20), tres aspectos parecen estar claros: por un lado, "la nueva mestiza" como categoría, como tipo, es tanto el punto final del cambio para mejorar la humanidad como su agente, de tal manera que cada una y cada uno puede ser individuo en la frontera, experimentarla y aprender la vida con un pluralismo de identidades. Por otro lado, el texto es una invitación directa para los lectores y las lectoras, que culmina en un llamamiento directo al lector específico y a la lectora específica (“you”). Se trata de una invitación a la experiencia y al acto: dos aspectos fundamentales para la realización de la utopía política de la humanidad transformándose para ser mejor. Finalmente, el libro es un medio de comunicación en un amplio sentido. Constituye la posibilidad tanto de expresión para que la autora se exprese, como para que cada uno de sus lectores y lectoras realice una experiencia mental y físico-corporal a través de la lectura.

\section{La materialidad del texto}

El texto subraya la naturaleza del libro, su existencia material, concretizada a través de la voz de quien lo lee, quien lo experimenta. Así, el concepto de la obra como obra de arte no se agota en el 
modo de representación de identidades alternativas, sino que revela su carácter específico (así como político) a través de sus posibilidades procesuales. En varios pasajes hay una insistencia en el carácter del texto como algo concreto, como objeto, pero un objeto vivo. El sexto capítulo contiene varias expresiones sobre el proceso de la escritura, la categoría del lector y del texto; se trata de un capítulo meta-poético. La instancia narrativa describe el libro en términos físicos:

In looking at this book that I'm almost finished writing, I see a mosaic pattern [...]. I can see the deep structure [...]. Too, I see the barely contained color threatening to spill over the boundaries of the object it represents [...]. I see a hybridization of metaphor [...]. The whole thing has had a mind of its own [...]. (Anzaldúa, 1999: 88)

Se hace hincapié en el aspecto performativo: “My 'stories' are acts encapsulated in time, 'enacted' every time they are spoken aloud or read silently. I like to think of them as performances and not as inert and 'dead' objects [...]" (Anzaldúa, 1999: 89). Y: "The ability of story (prose and poetry) to transform the storyteller and the listener into something or someone else [...]" (Anzaldúa, 1999: 88), “The writer, as shape-changer" (Anzaldúa, 1999: 88). En este punto podemos resumir que en el propio texto existe un concepto de la obra de arte como objeto vivo que supera, en cierta medida, el posicionamiento de su autora y que se caracteriza por su potencial de ser leído, lo cual significaría de nuevo un acto de experiencia conceptual vivida.

\section{Una comunicación intersubjetiva multidimensional}

En este mismo capítulo se manifiesta además la hibridez de géneros, que puede ser entendida como una yuxtaposición de diversos géneros dentro de la obra y una estética compuesta de variados estilos. ${ }^{14}$ Por ejemplo, se puede imaginar un estilo autobiográfico tanto en el género de la novela como en el género del ensayo o de la lírica. El efecto de tal estética es la posible dirección a "audiencias" diversas (Richardson, 1997). ${ }^{15}$ Se traduce como el intento de comunicar de esta manera el logro de satisfacer las diferentes dimensiones de experiencia de realidades individuales y colectivas. Aquí encontramos pasajes de estilo autobiográfico (la instancia narrativa recuerda momentos de su vida), pasajes de estilo ensayístico (leemos reflexiones sobre la esencia de la poesía y del proceso de escribir), pasajes de estilo dramatúrgico (la diosa Antigua está invocada de manera escénica), pasajes de estilo teórico (una teoría sobre el arte se desarrollada de manera argumentativa) y, finalmente, pasajes más bien líricos (instrumentos para escribir y momentos del proceso de escritura que son descritos a través de una retórica muy artística, sin comentarios a nivel de la instancia narrativa). Desde una perspectiva semántica, el capítulo está ligado a través de la isotopía de "escribir", no obstante, en cuanto a los estilos se fragmenta. Dada la situación de comunicación del texto expresada en el prefacio, ese pluralismo puede ser interpretado con respecto a los lectores y las lectoras. A partir de la premisa de la participación de la lengua en la construcción de realidades, los diferentes estilos del texto con sus diferentes estéticas se pueden interpretar como diferentes modos de crear un acceso a la realidad. En el acto de leer, entonces, los lectores y las lectoras experimentan el cambio de modos de existencia de las

\footnotetext{
${ }^{14}$ El concepto tradicional de "estilo" se refiere a categorías fijas dentro de la crítica y la historia literarias, como por ejemplo un estilo personal de un autor o una autora o también de un estilo histórico. Contrario a esta noción tradicional, aquí utilizamos el término para destacar el punto de vista del emisor y de la emisora del enunciado y sus varios modos de hablar. Lo utilizamos para describir variaciones en los modos de hablar que pueden cambiar incluso dentro de un párrafo y que representan formaciones de discursos diferentes. En este sentido, se asemejan a las 'voces' y a los géneros discursivos de Bajtín (1989).

${ }^{15}$ Richardson critica conceptos que postulan "a kind of archetypal reader" (32) y sugiere trabajar con el concepto de audience: "[...] a more flexible, heterogenous, comprehensive, and admittedly untidy category like that of "multiple and divided audiences”" (Richardson, 1997: 38). La combinación de la situación de comunicación entre autores y lectores y la estética de diferentes estilos en Borderlands/La frontera implica la utilización de tal concepto modificado.
} 
cosas. Se establece un acceso al fenómeno del arte transmitido, por ejemplo, a través de una teoría de arte, a través de una narración, a través de un dicho del abuelo o a través de un poema.

El análisis poetológico posibilita así concluir que Borderlands/La Frontera presenta un concepto de creación de la realidad que cuenta con un componente imaginativo, mediante palabras y textos; pero también con intensidad, dada la experiencia físico-corporal, a través de diferentes estilos o enunciados. ${ }^{16}$ Solo la unión de ambos aspectos constituye la condición de la realización del proyecto político que es importante tanto para el texto como para la autora histórica Gloria Anzaldúa.

\section{De cuerpos y espacios: del espacio textual al espacio físico-corporal}

Es un topos en la literatura de investigación y en los Chicano y Chicana Studies que los "Borderlands" de Gloria Anzaldúa representan al mismo tiempo un espacio físico, el del suroeste de los Estados Unidos; y un espacio metafórico, el de los "psychological borderlands, the sexual borderlands and the spiritual borderlands" (Anzaldúa, 1999: 19): "The borderlands are only in part geographic; instead the borderlands must be understood metaphorically, as a state of being and consciousness that is constantly being redefined" (Perales, 2013: 163). En Borderlands/La Frontera los borderlands se constituyen como un espacio de contacto cultural conflictivo. Por un lado, se mencionan las discriminaciones, injusticias y privaciones concretas que sus habitantes chicanos, los inmigrantes mexicanos ilegales y sus descendientes sufren a diferentes niveles. Por otro, el texto traduce este encuentro doloroso de dos espacios físico-lingüístico-culturales angloamericanos y mexicanos en la metáfora de la "herida abierta", a través de la cual la sangre de los dos mundos se mezcla y forma una tercera cultura: "a border culture" (Anzaldúa, 1999: 25; cursivas del original).

Este recurso a la imagen del cuerpo subraya la importancia de lo físico-corporal para la producción de los borderlands como un espacio en que los sujetos perciben y viven a través de su cuerpo. Además, destaca la tensión entre el énfasis en la discursividad de los borderlands, que prevalece en la totalidad del texto, y en sus características materiales, como el viento o la sequía, experimentadas físicamente por sus habitantes; así como su construcción discursiva, ya que obviamente los lectores y las lectoras perciben el espacio fronterizo mediante su propia discursividad. Esta tensión entre lo físicomaterial y lo discursivo remite a las preguntas de cómo se constituye concretamente el espacio de los borderlands en el texto y cómo se realiza la relación entre las prácticas sociales en el espacio material, los conceptos espaciales y las experiencias vividas del espacio que se traducen en una imaginación y metafórica espacial.

\section{Espacio y cuerpo desde una perspectiva fenomenológica}

Para acercarse a esta problemática es productivo recurrir al concepto fenomenológico del espacio del geógrafo francés Henri Lefebvre, quien en su obra La production de l'espace (1974) define el

\footnotetext{
${ }^{16}$ Nos referimos a una concepción del "enunciado" similar a la de las "voces" que Mijail Bajtín desarrolla en Teoría y estética de la novela (1989).

17 Véase el prefacio: “The actual physical borderland that I'm dealing with in this book is the Texas-U.S Southwest/Mexican border. The psychological borderlands, the sexual borderlands and the spiritual borderlands are not particular to the Southwest. In fact, the Borderlands are physically present wherever two or more cultures edge each other, where people of different races occupy the same territory, where under, lower, middle and upper classes touch, where space between two individuals shrinks with intimacy" (Anzaldúa, 1999: 19).
} 
espacio social como el producto y el proceso socio-dialéctico entre tres dimensiones: el espacio percibido, el espacio concebido y el espacio vivido. ${ }^{18}$ En esta triada se corresponden al espacio percibido prácticas espaciales como viajar, colonizar, trabajar la tierra. Con el espacio concebido se asocian las representaciones del espacio como el mapa o conceptos políticos y, por último, el espacio vivido (y sufrido) se relaciona con los espacios de representación como monumentos históricos, mitos, pero también con obras de arte e incluso textos literarios (Lefebvre, 2000: 42-43). Esta producción espacial se efectúa según el concepto fenomenológico de Lefebvre a través del cuerpo como órgano de percepción del sujeto (Lefebvre, 2000: 50). Es el cuerpo inseparable de la mente lo que produce el fenómeno del espacio en base a los estímulos visuales, acústicos, táctiles, olfatorios recibidos a partir de su ámbito social, material y mental. Desde una perspectiva poscolonial e interseccional cabe destacar la importancia del posicionamiento interseccional (Crenshaw, 1991; Quijano, 2000) de un sujeto dentro de una sociedad para su producción espacial. ${ }^{19}$ Este posicionamiento se configura en gran parte a través del cuerpo de un sujeto y su clasificación social en función de este cuerpo, observación particularmente valiente en contextos coloniales o poscoloniales que funcionan según un esquema binario (Fanon, 2002; Kipfer, 2007; Soazo, 2018). En este sentido, el posicionamiento interseccional influye en la producción espacial de un sujeto, mientras que al revés un espacio dado se inscribe igualmente en el sujeto y en su cuerpo.

Si se transfiere tal modelo sociológico a la cuestión de la producción del espacio por el texto, se puede decir que, en este último, dada su calidad de espacio de representación, se produce un espacio textual que amplía el espacio más allá de la mera representación. Asentado en la interfaz de las representaciones del espacio y de la imaginación espacial, se construye a nivel intratextual mediante la negociación de las tres dimensiones mencionadas (percibido, concebido, vivido), y se hace partícipe en la producción del espacio extratextual de sus lectores y lectoras. Al mismo tiempo, la producción del espacio textual obedece a las condiciones y normas de los géneros literarios. Dichas normas permiten, por ejemplo, en el caso de los textos narrativos, presentar múltiples producciones espaciales a través de las y los protagonistas y dirigir la mirada de las lectoras y los lectores a través de la perspectiva narrativa, mientras que los textos poéticos carecen de estos matices y posibilitan un acceso más inmediato al espacio producido en y por ellos.

A partir del prefacio, el primer capítulo y una selección de poemas, en el siguiente análisis nos interesará ver cómo Borderlands/La Frontera produce el espacio principalmente textual de los borderlands en dos planos: primero, en el de la relación entre las dimensiones de lo percibido, de lo concebido y de lo vivido (las prácticas espaciales, los conceptos espaciales y las imaginaciones espaciales) y segundo, en el de las estrategias textuales empleadas. Vista la hibridez genérica de Borderlands/La Frontera mencionada anteriormente, nos centraremos además en las (posibles) divergencias concernientes a las producciones del espacio y en la interacción entre las partes ensayísticas y las partes poéticas del texto. ¿En qué medida, por ende, la materialidad de los borderlands prima sobre su plano discursivo? ¿Cuál es la función específica de la comunicación por y mediante el cuerpo y la corporalidad?

\section{Dirigiendo la mirada de diferentes audiencias}

\footnotetext{
18 Véase "L'espace (social) est un produit (social)" (Lefebvre, 2000: 35).

19 A pesar de la procedencia marxista del modelo espacial de Lefebvre, este mismo tiene que ser decolonizado. Se debe completar su enfoque con el aspecto de la clase social y conceder más relevancia a los paradigmas de la colonialidad (Quijano, 2000) y de la interseccionalidad (Crenshaw, 1991; McCall, 2005) que enfatizan la importancia de las variables estructurales del sexo/género y la etnicidad/raza.
} 
El prefacio ("Preface to the First Edition") llama la atención de los lectores y las lectoras sobre la aparente dicotomía entre los borderlands físicos y los psicológicos o, en la terminología de Lefebvre, entre el espacio percibido y el espacio vivido (Anzaldúa, 1999: 19). El texto contempla lo físico-material no solo a raíz de la definición de la "Texas-U.S Southwest/Mexican border" (Anzaldúa, 1999: 19), sino como el elemento que más define los espacios fronterizos metafóricos que ocupa la nueva mestiza en calidad de "colonized people in our own territory" (Anzaldúa, 1999: 19) en el plano psicológico, sexual y espiritual, como chicana lesbiana que reclama su herencia indígena. A este contexto se le relaciona la idea del cuerpo asentado en el punto del contacto y destrozándose entre los lados de la frontera: "I have been straddling that tejas-Mexican border, and other all my life. It's not a comfortable territory to live in, this place of contradictions" (Anzaldúa, 1999: 19). Por lo tanto, subraya la importancia de "la madre naturaleza" como instancia que conecta el yo de la instancia narrativa con la tierra ("that anchored me to earth"), así como fuente de imágenes transformadas a su alrededor en metáforas para la toma de conciencia de la nueva mestiza: "mesquite flowering, the wind, Ehécatl, whispering its secret knowledge, the fleeting images of the soul in fantasy" (Anzaldúa, 1999: 19-20; cursivas del original). Por ende, el prefacio sugiere que el cuerpo del texto represente el resultado de la tentativa de la autora de materializar su imaginación en el mundo y en el papel —es decir traducirla en actos de resistencia y transformarla en un documento textual, darle cuerpo ("flesh") (Anzaldúa, 1999: 19-20). ${ }^{20}$ Este lenguaje del cuerpo y de la corporalidad anunciado en el prefacio, desarrollado a través de las partes ensayísticas y poéticas y anclado verbalmente en la naturaleza material - "grow roots that anchored me to the earth" (Anzaldúa, 1999: 19-20)—, condiciona la experiencia de lectura y favorece, por lo tanto, un acercamiento fenomenológico al espacio tal y como se produce en el texto, es decir, un espacio percibido, concebido y vivido a través del cuerpo humano.

¿Cómo se configura la producción del espacio textual en esta obra? El primer capítulo de Borderlands / La Frontera, "The Homeland, Aztlán / El otro México", sirve de exposición a la pregunta del espacio de contacto de los borderlands. Se concentra en la producción de los borderlands como un espacio históricamente azteca, mestizo y mexicano anexionado brutalmente por los angloamericanos, resultado de la dominación y la explotación por los anglos del chicano y del inmigrante ilegal mexicano y, particularmente, de las mujeres chicanas y mexicanas. Además de las dimensiones de las prácticas espaciales y de los espacios de representación, se destaca aquí la conceptualización de los borderlands. "The Homeland, Aztlán / El otro México" consta de tres partes tituladas "El otro Mexico", "El destierro / The Lost Land", "El cruzar del mojado/Illegal Crossing", que corresponden a las distintas etapas de argumentación espacial: la primera parte plantea la situación actual de la frontera artificial que fractura el espacio cuya pertenencia histórica corresponde a los aztecas y mestizos; la segunda, plasma el destierro brutal de los mexicanos y mestizos por los invasores anglos; la tercera, describe la migración mexicana contemporánea hacia el norte como un retorno a la tierra ancestral.

Acorde con el carácter genéricamente híbrido de la obra, las largas porciones ensayísticas son introducidas o interrumpidas por poemas y citas de intertextos. Estos tienen una función particular concerniente a la producción textual del espacio de los borderlands (físicos) como espacio fronterizo y, por esto, de contacto cultural, ya que ilustran ante todo la dimensión del espacio vivido de Lefebvre: traducen, según nuestra hipótesis, la experiencia del espacio en la imagen/metáfora del cuerpo humano al mismo tiempo que insisten en la configuración fundamental del espacio de los borderlands por las culturas ancestrales indígenas y mestizas. Por lo contrario, en las partes ensayísticas predominan las dimensiones de las prácticas espaciales y las representaciones políticas del espacio fronterizo. Al

\footnotetext{
20 "Books saved my sanity, knowledge opened the locked places in me taught me how to survive and then how to soar. $\mathrm{La}$ madre naturaleza succored me, allowed me to grow roots that anchored me to the earth. My love of images — mesquite flowering, the wind, Ehécatl, whispering its secret knowledge, the fleeting images of the soul in fantasy- and words, my passion for the daily struggle to render them concrete in the world and on paper, to render them flesh, keeps me alive" (Anzaldúa, 1999: 19-20; cursivas del original).
} 
vincularse un discurso de estilo historiográfico con uno de estilo autobiográfico, el capítulo pondera el entrelazamiento entre Historia (Histoire) e historias personales (bistoire) (Glissant, 1989: 264).

\section{El estilo lírico: teniendo puentes entre espacios percibidos y espacios vividos}

Para corroborar la observación desarrollada en la última parte del apartado anterior, basamos el siguiente análisis de la interacción genérica en la producción textual de los borderlands en el subcapítulo "El otro México". Dos epígrafes, tomados de una canción del grupo norteño Los Tigres del Norte ${ }^{21}$ y de una obra historiográfica, ${ }^{22}$ introducen esta parte. Estos remiten concretamente al México perdido, el borderland actual, que se reconstruye como "otro México" gracias a los inmigrantes mexicanos y latinoamericanos: "El otro México que acá hemos construido / [...] / es el esfuerzo de todos nuestros hermanos y latinoamericanos" y que corresponde a la tierra antigua de los Aztecas, Aztlán (Anzaldúa, 1999: 23). Asimismo, hacen referencia al espacio concebido como "territorio nacional" (Anzaldúa, 1999: 23), a la práctica espacial de la migración como reapropiación del espacio al mismo tiempo que acentúan la historia indígena ancestral del espacio de los borderlands (en contraste con una visión de esta tierra como "terra nullium").

Si los dos epígrafes ya dirigen la mirada de los lectores y las lectoras hacia la anticipación del espacio que el capítulo producirá, el poema escrito por Anzaldúa que los sigue destaca por su configuración de la frontera y del espacio fronterizo (Anzaldúa, 1999: 23-25). Incluso la superficie del poema caligrama, su presentación visual ondulatoria, semejante a formas naturales como el mar o la montaña, pone de relieve su estrecha relación con lo físico y el paisaje. Esta relación se refleja en la posición física del yo que habla desde la frontera entre tierra y mar: "I stand at the edge where earth touches ocean / where the two overlap / a gentle coming together / at other times and places a violent clash" (23). El poema escenifica la mirada hacia la cerca, el "border fence", desde el lado estadounidense. Se trata sobre todo de una experiencia sensual. A través de los ruidos que el yo escucha — "el llorido del mar" (24; cursivas del original), "the gulls' shrill cry of hunger" (24)—, los olores — "the tangy smell of the sea seeping into me" (24)—; las impresiones visuales — "stark silhouette of houses gutted by waves", "miro el mar atacar la cerca en Border Field Park" (24; cursivas del original) — y el tacto - "Under my fingers a feel the gritty wire rusted by 139 years of the salty breath of the sea" (24), "I press my hand to the steel curtain" (24)—; el yo percibe y vive la frontera de manera física y después mental. La materialidad de la frontera, experimentada por el cuerpo, se transforma por la experiencia mental verbalizada del sujeto escritor en un texto que se puede leer, una experiencia que se puede vivir a través de la lectura.

Asimismo, la voz lírica compara el cuerpo herido por la cerca con la superficie sin sutura de la naturaleza: "But the skin is seamless. / The sea cannot be fenced, / el mar does not stop at borders" (Anzaldúa, 1999: 25), para al final definir el yo como un "Puente tendido / del mundo gabacho al del mojado, / lo pasado me estira $p a$ ' 'tràs / y lo presente pa' delante" (25; cursivas del original) a nivel figurado y concreto. Sin embargo, para retornar a la cuestión de las dimensiones espaciales predominantes en el poema, este no solo plasma la experiencia física de la frontera material y su transposición en la imagen de México como un cuerpo rajado ("me raja me raja"), sino que concibe el espacio fronterizo como el resultado de un solapamiento físico a veces apacible y frecuentemente violento — “overlap", "a gentle coming

\footnotetext{
${ }^{21}$ En el primer epígrafe se lee: "El otro México / que aquí hemos construido / en este suelo que ha sido / territorio nacional / es el esfuerzo/ de todos nuestros hermanos / y latinoamericanos / que han sabido progresar". El epígrafe utilizado por Anzaldúa modifica el texto de la canción "El otro México" ligera, pero significativamente: "El otro Mexico / que acá hemos construido / el espacio es lo que ha sido / territorio nacional. / Este es el esfuerzo de todos nuestros hermanos / y latinoamericanos que ban sabido / progresar" (Anzaldúa, 1999: 23; cursivas del original).

${ }^{22}$ Esta segunda citación está, según Borderlands/La Frontera, tomada de Jack D. Forbes: Aztecas del Norte 1973. Comienza con las siguientes palabras: "The aztecas del norte compose the largest single tribe... [...]" (Anzaldúa, 1999: 23).
} 
together / at other times and [...] a violent clash" (23). A fin de plasmar este concepto, se emplea de nuevo una imagen del paisaje físico-material, es decir, el juego del solapamiento oscilante entre tierra y mar mientras que la línea, el borde de la frontera, parece innatural y artificial como un corte.

\section{El estilo ensayístico como mediación entre los espacios percibidos y concebidos}

A continuación, la parte en prosa del capítulo retoma la metafórica corporal del poema para definir la frontera ya nombrada en el poema con el término inglés "open wound": se trata de "una herida abierta where the Third World grates against the first and bleeds. And before a scab forms it hemorrhages again, the lifeblood of two worlds merging to form a third country —a border culture" (Anzaldúa, 1999: 25). Sigue refinando la dimensión conceptual de la frontera como borde y espacio a la vez bajo un ángulo político ya que, según el texto en prosa, las fronteras tienen el objetivo de construir y reificar diferencias entre sujetos mientras que los borderlands constituyen un espacio ambivalente y transitorio. ${ }^{23}$

De acuerdo con el texto, las jerarquías y tensiones racistas y machistas determinan este espacio fronterizo en todas sus dimensiones, ya sean estas las prácticas espaciales, las representaciones espaciales o los espacios de representación. El discurso acentúa en particular la dimensión de las prácticas sociales en los borderlands, que definen la relación entre los invasores anglos y las poblaciones racializadas/étnicamente marcadas del suroeste de EE.UU. (Anzaldúa, 1999: 26). Como demuestra el episodio de estilo autobiográfico intercalado con el discurso ensayístico, las relaciones de poder conducen a prácticas espaciales antagonistas: mientras que los anglos deportan a los chicanos, sean ciudadanos estadounidenses o no, estos retornan como inmigrantes ilegales a su propia tierra (Anzaldúa, 1999: 26-27). Al mismo tiempo, el discurso historiógrafo presenta los borderlands como un espacio profundamente mexicano, puesto que históricamente acogió poblaciones mexicanas en varias ocasiones: se trata de la tierra ancestral de las primeras naciones indígenas de la que sus descendientes aztecas migraron hacia Teotihuacán alrededor del 1000 a.C. y a la que después de la conquista, españoles, indígenas y mestizos retornaron a partir del siglo XVI (Anzaldúa, 1999: 26-27).

A la luz de estas observaciones se puede constatar que: (1) los epígrafes, poemas e intertextos sirven a menudo para metaforizar y traducir el espacio material en la imagen del cuerpo humano herido, así como para dirigir a través de textos artísticos una y otra vez la mirada de los lectores y las lectoras hacia la estrecha vinculación histórica de los borderlands con las culturas aztecas y mestizas. La dinámica textual lleva al sujeto lector del espacio percibido hacia el espacio vivido según las categorías fenomenológicas de Lefebvre. A diferencia de esta concentración en la dimensión del espacio vivido y de sus productos culturales, las partes en prosa de estilo historiógrafo y autobiográfico se encuentran más en relación con las prácticas espaciales y sus incidencias a nivel colectivo e individual. Además, las partes en prosa se dedican a la conceptualización de la noción de la frontera y del espacio fronterizo bajo un ángulo político. Análogamente, según las categorías fenomenológicas de Lefebvre, la dinámica textual también lleva al sujeto lector del espacio concebido hacia el espacio percibido. Esta conceptualización sirve de puente entre la experiencia física individual y la subsecuente metaforización de la frontera, por un lado, y las prácticas espaciales a nivel político y colectivo por otro lado.

Por ende, el texto envuelve a sus lectores y lectoras en un movimiento tanto argumentativo como material. Mediante el poema introductorio el sujeto lector entra en contacto con la experiencia física metaforizada de los borderlands del sujeto escrito/escritor, ${ }^{24}$ mientras que en las partes en prosa se

\footnotetext{
23 "Borders are set up to define the places that are safe and unsafe, to distinguish us from them. A border is a dividing line. A narrow strip along a steep edge. A borderland is a vague and undetermined place created by the emotional residue of an unnatural boundary. It is in a constant state of transition. The prohibited and the forbidden are its inhabitants" (Anzaldúa, 1999: 25).

${ }^{24}$ Con la noción de 'sujeto escrito/escritor' nos referimos al carácter doble del sujeto del enunciado y de la enunciación.
} 
le recuerda constantemente a la audiencia la pertenencia cultural de los borderlands. En los intertextos intercalados el sujeto lector se ve enfrentado a las experiencias colectivas concretas, pero menos accesibles debido al género literario.

A través de la experiencia físico-corporal del sujeto escrito/escritor étnicamente marcado, que se transmite y traduce en un texto verbal ante todo en las partes líricas, se constituye un acceso corporal del sujeto lector al espacio textual. Este espacio textual lo coproduce el sujeto lector en el acto mismo de la lectura - en función de su propio posicionamiento interseccional y cultural.

\section{El espacio fronterizo como un espacio de diálogo intersubjetivo}

Si volvemos a las preguntas iniciales de la (auto)reflexión poetológica del texto en su cualidad de obra de arte productora de espacios textuales, se puede constatar que Borderlands/La Frontera: The New Mestiza brinda a sus lectores y lectoras no solo una reflexión sobre los espacios físicos y metafóricos de la frontera, sino que va más allá y formula también una reflexión sobre la necesidad y las posibilidades de la intersubjetividad en el mundo 'real', así como en las experiencias de escritura/lectura. Tanto en las porciones del texto dedicadas expresamente a la producción de un espacio textual referencial como en los capítulos autoreflexivos, se efectúa un movimiento argumentativo que atraviesa las fronteras entre los polos de lo individual y lo colectivo, entre lo físico-corporal y lo metafórico. En este movimiento introducido en el prefacio como principio fundamental de la obra destaca el dinamismo que lleva del "yo" individual de la "border woman" a la invitación para audiencias concretas —el sujeto lector del "you" - al diálogo con el colectivo de "las new Mestizas" (Anzaldúa, 1999: 20) ${ }^{25}$ y culmina finalmente en un movimiento de oscilación que estructura la obra entera en múltiples planos.

La pluralidad de géneros y estilos literarios genera en consecuencia diferentes modos de enunciación, conectados con ciertas temáticas, que permiten al texto/sujeto-escritor mostrar el mismo fenómeno bajo varios ángulos y al sujeto lector experimentar esta variedad de facetas y forjar su propia producción individual de los borderlands, físicos y —estrechamente entrelazado-metafóricos. De igual modo, se podría decir que, en cierta medida, es el propio texto de Borderlands/La Frontera que produce otro espacio, virtual en su entorno, un espacio de contacto intersubjetivo en el que el sujeto lector y el sujetoescrito/escritor se encuentran a través de la experiencia material y corporal en la lectura del discurso.

La obra marca un contrapunto en relación con concepciones posmodernas optimistas en cuanto a la categoría de "pluralidades discursivas". Se entiende una idea de la obra de arte como objeto que representa una experiencia vivida y que puede ser experimentada de manera físico-corporal. Además, Borderlands/La Frontera no solo representa realidades plurales, sino que forma una totalidad heterogénea, pero no fragmentada. En este punto, se destaca igualmente de proyectos de arte fronterizos recientes, 'posposmodernos', tal como la instalación de realidad virtual Carne y Arena de González Iñárritu; y de los productos de las industrias culturales, tal como películas que hacen espectáculos del cruce peligroso de la frontera hoy en día fuertemente militarizada. A pesar de su voluntad de hacer perceptible el espacio sufrido del Otro, esta simulación del cruce fronterizo se presta a un mero consumo de experiencias marginales. A través de su propia estructura, el texto de Anzaldúa les da a sus audiencias, mayormente privilegiadas, la ilusión de ponerse físicamente en la piel del Otro cuando en realidad lo obvia. En cambio, la construcción poetológica y espacial de Borderlands/La Frontera pone en escena una oferta de diálogo intersubjetivo, dentro de la cual el posicionamiento del Otro es imprescindible.

\footnotetext{
25 "Today we ask to be met halfway. This book is our invitation to you-from the new mestizas" (Anzaldúa, 1999: 20).
} 


\section{Bibliografía}

ÁBrego, Perla (2011), "La frontera como sistema simbólico en la literatura mexicana contemporánea”, en Revista Surco Sur, vol. 2, n. ${ }^{\circ}$ 3, pp. 47-52. DOI: <http://dx.doi.org/10.5038/2157-5231.2.3.13>.

Aldama, Arturo J. (2001), Dismpting Savagism: Intersecting Chicana/o, Mexican Immigrant, and Native American Struggles for Self-Representation. Durham, Duke University Press, pp. 95-128. DOI: <https://doi.org/10.1515/9780822380016-006>.

AnZaldúA, Gloria E. (1999) [1987], Borderlands/La Frontera: The New Mestiza. San Francisco, Aunt Lute Books.

BajTín, Mijail (1989), Teoría y estética de la novela.. Kriukova, Helena S. y Vicente Cazcarra (trads.), Madrid, Taunus.

BANDAU, Anja (2020), "Borderlands revisited: La frontera norte in contemporary Mexican fiction”, en iMex. México Interdisciplinario. Interdisciplinary Mexico, año 9 vol. 1, n. ${ }^{\circ}$ 17, pp. 19-35.

BArrera, Cordelia (2020), "Accessing Gloria Anzaldúa through Utopia”, en Cantú-Sánchez, Margaret; León-Zepeda, Candace de; Cantú, Norma E. (eds.), Teaching Gloria E. Anzaldúa: Pedagogy and Practice for Our Classrooms and Communities. Tucson, University of Arizona Press, pp. 47-58. DOI: $<$ https://doi.org/10.2307/j.ctv1595m67>.

BORNSTEIN-GÓMEZ, Miriam (2010), "Gloria Anzaldúa: Borders of Knowledge and (re)Signification”, en Confluencia, vol. 26, n. 1 , pp. 46-55.

Castillo, Debra y María Socorro Tabuenca (eds.) (2002), Border Women. Writing from La Frontera. Minneapolis, University of Minnesota Press.

Castillo, Debra (2006), "Anzaldúa and Transnational American Studies", en Publications of the Modern Language Association of America, vol. 121, n. ${ }^{\circ}$ 1, pp. 260-265. DOI: <https://doi.org/10.1632/003081206X129819>.

CRENSHAW, Kimberlé W. (1991), "Mapping the Margins: Intersectionality, Identity Politics, and Violence Against Women of Color", en Stanford Law Review, vol. 43, n. ${ }^{\circ}$ 6, pp. 1241-1299. DOI: $<$ https://doi.org/10.2307/1229039>.

DAHMS, Betsy (2021), "Imaginar un hogar: desplazamiento psicológico y su relación con la imaginación en tres obras de Gloria Anzaldúa”, en Gallo González, Danae; Leuzinger, Mirjam; Dolle, Verena (eds.), Hispan@s en el mundo: Emoción y desplazamiento. Berlin/ Boston, De Gruyter, pp. 17-36. DOI: $<$ https://doi.org/10.1515/9783110727555-002>.

ETTE, Ottmar; Wirth, Uwe (eds.) (2014), Nach der Hybridität: Zukïnfte der Kulturtheorie (Pointe 11). Berlin, Tranvía.

FANON, Frantz (2002) [1961], Les damnés de la terre. Paris, La Découverte.

Garcia Canclini, Nestor (1990), Culturas hibridas. Estrategias para entrar y salir de la modernidad. Ciudad deMéxico, Grijalbo.

GarZA, Elisa A. (2003), "Chicana Lesbianism and the Multigenre Text", en Torres, Lourdes (ed.), Tortilleras: Hispanic and U.S. Latina Lesbian Expression. Philadelphia, Temple University Press, pp. 196-210.

GEWECKE, Frauke (2012), "De espacios, fronteras, territorios: topografías literarias de la Frontera Norte (México)", en Iberoamericana, vol. 12, n. ${ }^{\circ}$ 46, pp. 111-127.

GIMÉNEZ, Gilberto (2007), "La frontera norte como representación y referente cultural en México", en Cultura y Representaciones Sociales, vol. 2, n. ${ }^{\circ}$ 3, pp. 17-34.

GLISSANT, Édouard (1989) [1981], Le discours antillais. Paris, Seuil. 
HEIDE, Markus (2004), Grenఇüberschreibungen. Chicano-Erzählliteratur und die Inszenierung von Kulturkontakt. Heidelberg, Winter.

HeIDE, Markus (2021), “Learning from Las Vegas': Border Aesthetics, Disturbance, and Electronic Disobedience. An Interview with Performance Artist Ricardo Dominguez", en Journal of Borderlands Studies, vol. 36, n. ${ }^{\circ}$ 1, pp. 49-57. DOI: <https://doi.org/10.1080/08865655.2018.1490197>.

Keating, AnaLouise (1996), Women Reading Women Writing: Self-Invention in Paula Gunn Allen, Gloria Anzaldua and Audre Lourde. Philadelphia, Temple University Press.

Keating, AnaLouise (2005) (ed.), Entre Mundos, Among Worlds: New Perspectives on Gloria E. Anzaldúa. New York, Palgrave Macmillan.

KIPFER, Stefan (2007), "Fanon and Space. Colonization, Urbanization, and Liberation from the Colonial to the Global City", en Environment and Planning D. Society and Space, vol. 25, n. ${ }^{\circ}$ 4, pp. 701-726. DOI: $<$ https://doi.org/10.1068/dkipfer>.

LANGER, Daniela (2007), “Autobiografie”, en Anz, Thomas (ed.), Handbuch Literatumissenschaft. Gegenstände Konzepte - Institutionen (2). Methoden und Theorien. Stuttgart/ Weimar, Metzler, pp. 179-187.

LefeBVRE, Henri (2000) [1974], La production de l'espace. Paris, Anthropos.

LejEunE, Philippe (2001) [1991], Le pacte autobiographique. Paris, Seuil.

MCCALL, Leslie (2005), “The complexity of intersectionality”, en Signs, vol. 30, n. o 3, pp. 1771-1800.

NuÑo, Anthony (2020), "Irreverent Pedagogies: Las palabras sabias y poderosas de Gloria Anzaldúa”, en Cantú-Sánchez, Margaret; León-Zepeda, Candace de; Cantú, Norma E. (eds.), Teaching Gloria E. Anzaldua: Pedagogy and Practice for Our Classrooms and Communities. Tucson, University of Arizona Press, pp. 47-58. DOI: < https://doi.org/10.2307/j.ctv1595m67>.

Perales, Monica (2013), "On Borderlands/La Frontera: Gloria Anzaldúa and Twenty-Five Years of Research on Gender in the Borderlands", en Journal of Women's History vol. 25, n. ${ }^{\circ}$ 4, pp. 163-173. DOI: <https://doi.org/10.1353/jowh.2013.0047>.

PRATT, Mary Louise (1992), Imperial Eyes: Travel Writing and Transculturation. London, Routledge.

Qujano, Anibal (2000), "Colonialidad y clasificación social”, en Journal of World-Systems Research vol. 2, Special Issue: Festschrift for Immanuel Wallerstein, Part I, pp. 342-386.

RADLWIMMER, Romana (2020), "Transborder Matters: A Conceptual Approach", en iMex. México Interdisciplinario. Interdisciplinary Mexico, año 9, vol. 1, n. ${ }^{\circ}$ 17, pp. 8-17.

Richardson, Brian (1997), “The Other Reader's Response: On Multiple, Divided, and Oppositional Audiences", en Criticism, vol. 39, n. ${ }^{\circ}$, pp. 31-53.

SOAzo AhumadA, Christian (2018), "Espacio colonial y aesthesis soberana: praxis decolonial desde la experiencia vivida del negro según Fanon", en Pléyade. Revista de bumanidades y ciencias sociales, vol. 21, Enero-junio, pp. 93-117. DOI: <http://dx.doi.org/10.4067/S0719-36962018000100093>.

STEHN, Alexander V. y Mariana ALESSANDRI (2020), "La Mexicana en la Chicana: The Mexican Sources of Gloria Anzaldúa’s Inter-American Philosophy”, en Inter-American Journal of Philosophy, vol. 1, n. 11, pp. 44-62.

Socorro Tabuenca, María (1995), "Viewing the Border: Perspectives from 'The Open Wound", en Discourse, vol. 18, n. $\quad 1-2$, pp. 146-168.

VILA, Pablo (2000), "La teoría de frontera versión norteamericana. Una crítica desde la etnografía", en Grimson, Alejandro (ed.), Fronteras, naciones e identidades. La periferia como centro. Buenos Aires, Ediciones Ciccus La Crujía, pp. 99-120. 